\title{
Efeito sinérgico entre Digoxina e Cisplatina para o tratamento do câncer de colo uterino
}

\author{
Pereira D. G*, Salgado M. A. R., Barbosa L. A \\ Departamento de Bioquímica Celular, Universidade Federal de São João Del-Rei, Divinópolis/MG \\ *e-mail: duanegpereira@hotmail.com
}

\section{Resumo}

Atualmente vários estudos demonstram interessantes efeitos antitumorais com glicosídeos cardíacos para uma série de culturas celulares. A quimioterapia para o tratamento de câncer de colo uterino utiliza de compostos derivados de platina, e o mais amplamente utilizado é a cisplatina. Porém, a cisplatina causa efeitos colaterais graves, como a mielossupressão, náuseas, ototoxicidade, nefrotoxicidade e fadiga, além de possuir uma baixa taxa de resposta para este tipo de câncer. $\mathrm{O}$ objetivo deste trabalho foi avaliar o possível efeito citotóxico e antiproliferativo do tratamento combinado de digoxina e cisplatina em células de câncer de colo uterino, bem como verificar se esse tratamento está desenvolvendo seus efeitos mediados pela atividade $\mathrm{Na}, \mathrm{K}$-ATPase e elucidar a possível via de sinalização que estaria desencadeando o processo. Para determinação da citotoxicidade foram realizados ensaio de viabilidade celular (MTT) e para avaliação da proliferação celular foi realizado o ensaio com azul de tripan. Realizou-se a avaliação da atividade da Na,K-ATPase e a utilização de inibidores específicos para a verificação do efeito de ERK1/2 e Src sobre a proliferação celular. Os resultados obtidos demonstraram que o pré-tratamento com $1 \mathrm{nM}$ digoxina foi capaz de potencializar o efeito citotóxico e a atividade antiproliferativa de $1 \mu \mathrm{M}$ cisplatina. Esses tratamentos não foram capazes de modular a atividade da Na,K-ATPase e a utilização do inibidor para Src PP2 foi capaz de bloquear o efeito sinérgico entre $1 \mathrm{nM}$ digoxina e $1 \mu \mathrm{M}$ cisplatina. Como digoxina e cisplatina já são drogas utilizadas clinicamente este estudo abre possibilidades para ensaios clínicos com tratamento combinado, possibilitando um tratamento mais eficaz com menor toxicidade e efeitos colaterais para o paciente.

Palavras-chave: digoxina, cisplatina, Na,K-ATPase, ERK1/2 e MAPK. 
Apoio financeiro: CNPq e FAPEMIG. 